\title{
Blurring past and present: Using false memory to better understand false hearing in young and older adults
}

\author{
Eric Failes ${ }^{1}$ (D) - Mitchell S. Sommers ${ }^{1} \cdot$ Larry L. Jacoby $^{1}$ \\ Published online: 15 July 2020 \\ (C) The Psychonomic Society, Inc. 2020
}

\begin{abstract}
A number of recent studies have shown that older adults are more susceptible to context-based misperceptions in hearing (Rogers, Jacoby, \& Sommers, Psychology and Aging, 27, 33-45, 2012; Sommers, Morton, \& Rogers, Remembering: Attributions, Processes, and Control in Human Memory [Essays in Honor of Larry Jacoby], pp. 269-284, 2015) than are young adults. One explanation for these age-related increases in what we term false hearing is that older adults are less able than young individuals to inhibit a prepotent response favored by context. A similar explanation has been proposed for demonstrations of age-related increases in false memory (Jacoby, Bishara, Hessels, \& Toth, Journal of Experimental Psychology: General, 134, 131-148, 2005). The present study was designed to compare susceptibility to false hearing and false memory in a group of young and older adults. In Experiment 1, we replicated the findings of past studies demonstrating increased frequency of false hearing in older, relative to young, adults. In Experiment 2, we demonstrated older adults' increased susceptibility to false memory in the same sample. Importantly, we found that participants who were more prone to false hearing also tended to be more prone to false memory, supporting the idea that the two phenomena share a common mechanism. The results are discussed within the framework of a capture model, which differentiates between context-based responding resulting from failures of cognitive control and context-based guessing.
\end{abstract}

Keywords False hearing $\cdot$ False memory $\cdot$ Speech perception $\cdot$ Context effects $\cdot$ Aging

\begin{abstract}
Although early conceptualizations of memory and perception considered both to be veridical recordings of events and sensations that occurred, it is now clear that these processes are influenced by prior knowledge and expectations, and often reflect a reconstruction of the experienced events (Alba \& Hasher, 1983; Bartlett, 1932; Bugelski \& Alampay, 1961). One of the most powerful demonstrations of such active reconstruction in both memory and perception are cases in which our expectations result in either false memories or false perceptionsincorrectly remembering or perceiving events based on expectations. The study of false memory dates back at least to the publication of Bartlett's (1932) book Remembering, in which he showed that participants' memory for the Native American folk story "The War of the Ghosts" became distorted to more
\end{abstract}

Eric Failes

e.failes@wustl.edu

1 Department of Psychological and Brain Sciences, Washington University in St. Louis, 1 Brookings Dr., Campus Box 1125, St. Louis, MO 63130, USA closely resemble their own experiences. For example, although the story describes two men hunting seals, participants often recalled that the two men were fishing. The history of false perception research extends back even further, with hundreds of studies describing visual illusions being published before the start of the 20th century (see Coren \& Girgus, 1978).

One framework that has been useful for understanding the mechanisms mediating both false memory and false perception is the process dissociation framework (Jacoby, 1991). As applied to memory and perception, the process dissociation framework posits that individual memories and perceptual experiences are determined by both controlled and automatic processes. The relative importance of controlled and automatic processes in determining perceptual or memorial experiences can be established by having conditions in which the two function in concert as well as those in which they are in opposition. For example, Jacoby, Bishara, Hessels, and Toth (2005, Experiment 2) applied the process dissociation framework to understand the mechanisms underlying one type of false memory. In their study, young and older adult participants first studied a list of semantically related cue-target word pairs (e.g., knee-bend). Following the study phase, participants were 
again shown the studied word pairs, but in each case only a fragment of the second word in each pair was shown (e.g., $k n e e-b \_n \_$). Participants were tasked with verbally completing the fragment with the word from the study phase and judged their confidence in their answer by indicating whether they remembered the reported word from the study phase, whether the reported word simply seemed familiar, or whether they were guessing. The critical manipulation was that immediately prior to viewing the cue-fragment pair, a prime was briefly presented on-screen. The prime was either the word from the study phase that correctly completed the fragment (congruent condition; e.g., bend), an unstudied word that was semantically related to the cue and could complete the fragment (incongruent condition; e.g., bone), or a string of ampersands (baseline condition; $\& \& \& \& \&)$, introducing helpful, misleading, or no bias, respectively. Jacoby et al. suggested that successful retrieval of the study phase word indexed controlled processing, whereas responses based on the prime indexed automatic processes. In the congruent condition, reliance on either controlled or automatic processing would lead to a correct response (bend). However, in the incongruent condition, correct responding required participants to resist the automatic process (bone) and respond based on controlled processing (i.e., retrieval from the study phase).

Although both young and older adults in the study by Jacoby et al. (2005, Experiment 2) produced false memories (e.g., responding with the prime word bone rather than the studied word bend), older adults were more likely to produce such false memories and were more likely to characterize these false memories as "remembered" than were young adults. This difference was found despite equating performance in the baseline condition for young and older adults by presenting the cue-target pairs for a longer duration for older adults in the study phase. Jacoby et al. concluded that the age differences in performance in the incongruent condition were due to differences in cognitive control - an inability to resist an automatic response - rather than a difference in memory per se. The Jacoby et al. results therefore supported previous findings (Hay \& Jacoby, 1999; Jacoby, 1999; see Jacoby \& Rhodes, 2006) suggesting that, relative to young adults, older adults have an increased reliance on automatic rather than controlled processing as a basis for responding.

Rogers, Jacoby, and Sommers (2012, Experiment 2) conducted an analogous experiment examining the relative contributions of automatic and controlled processes to speech perception. Young and older adult participants first studied semantically related, cue-target word pairs (e.g., barn-hay) presented both visually and aurally. Once participants had learned the word pairs, they completed a speech perception task in which two words were presented aurally, the first word in the clear and the second word in background noise. The word pairs were either the same as those learned in the study phase (congruent condition; e.g., barn-hay), a cue from the study phase paired with a word differing from the learned target word by a single phoneme, known as a phonological neighbor (incongruent condition; e.g., barn-pay), or a pair of novel, unrelated words (baseline condition; e.g., cloud-fun). The signal-to-noise ratio (SNR) for the second word in the pair was set individually such that each participant achieved approximately $50 \%$ accuracy in the baseline condition. Participants were tasked with saying aloud the word they heard in the background noise and judging their confidence that they had actually heard the word in noise on a $0 \%-100 \%$ scale.

In this case, controlled processes indexed participants' ability to respond on the basis of sensory information (the acoustic signal) and automatic processes indexed their reliance on the semantic relationship between the two words. As in the memory experiment by Jacoby et al. (2005), using either automatic or controlled processes as a basis for responding would lead to a correct response in the congruent condition. However, in the incongruent condition, reliance on automatic processes would lead to an incorrect response (e.g., reporting having heard hay when pay was actually presented). Rogers et al. (2012) used the term false hearing to refer to responses based on context in the incongruent condition that were accompanied by high levels of confidence. As expected, baseline performance was approximately $50 \%$ in both groups, indicating that overall audibility was equated for young and older adults. Similar to the Jacoby et al. (2005, Experiment 2) findings with regard to false memory, both young and older adults experienced false hearing, but older adults were more prone to false hearing than were young adults. Additionally, older adults attributed higher confidence ratings to those incorrect responses than did young adults. Together, these findings indicate an age-related increase in responding based on automatic rather than controlled processes.

In a follow-up experiment, Sommers, Morton, and Rogers (2015, Experiment 2) demonstrated that the semantic context provided by natural speech also influences perception of individual words. Sommers et al. had young and older adults identify sentence-final words in background noise. The target words were either highly predicted by the preceding sentence (congruent condition; e.g., The shepherd watched his sheep), a phonological neighbor of the word predicted by context (incongruent condition; e.g., The shepherd watched his sheath), or were unpredictable from the preceding sentence (baseline condition; e.g., Paul heard they asked about the sheep). As in the study by Rogers et al. (2012, Experiment 2), the SNR was set individually such that each participant achieved approximately $50 \%$ accuracy in the baseline condition, and all participants made a $0 \%-100 \%$ confidence judgement after identifying each target word.

The results of Sommers et al. (2015, Experiment 2) provided a conceptual replication of both Jacoby et al. (2005, Experiment 2) and Rogers et al. (2012, Experiment 2). Participants correctly identified in excess of $90 \%$ of target words in the congruent condition, but frequently misheard the target words in the incongruent condition as the contextually predicted word (e.g., 
responding sheep when sheath was actually presented), demonstrating the ability of automatic responses - in this case, supported by the preceding sentence context - to override available sensory information. Furthermore, as in the studies by Jacoby et al. and Rogers et al., older adults were more prone to context-based errors and were more confident in those errors than were young adults, with older adults reporting maximum confidence in cases of false hearing - which the authors referred to as dramatic false hearing-four times as frequently as young adults (.16 versus .04). These findings further supported the idea that older adults are more reliant on automatic processes as a basis for responding than are young adults. Furthermore, as in the study by Rogers et al., performance in the baseline condition was equated between older and young adults by individually setting the SNR, meaning that age differences observed in the congruent and incongruent conditions could not be attributed to differences in hearing acuity.

In summary, both false memories (Jacoby et al., 2005) and false perceptions (Rogers et al., 2012; Sommers et al., 2015) can be accounted for within the process dissociation framework. Specifically, when automatic and controlled processes are placed in opposition, responding based on the former can lead to errors in memory and perception. Furthermore, age-related increases in both false memory and false hearing are consistent with greater reliance on automatic processing by older compared with young adults (Hay \& Jacoby, 1999; Jennings \& Jacoby, 1997). In the present study, we examined whether similar mechanisms mediate false memory and false perception by obtaining measures of both phenomena within the same individuals. In Experiment 1, we attempted to replicate the Sommers et al. (2015) finding of robust levels of false hearing in young and older adults. In Experiment 2, we used the memory test from Jacoby et al. (2005, Experiment 2) to assess susceptibility to false memory in the same group of young and older adults and compared this to their susceptibility to false hearing from Experiment 1. Finding that individual differences in false memory predict individual differences in false hearing would suggest that the two phenomena may, at least in part, arise from mechanisms that are not specific to either memory or perception, but instead reflect the operation of more global cognitive control mechanisms. This finding would be critical for developing a unified theory to explain context-based errors encompassing both false memory and false perception.

\section{Experiment 1}

\section{Materials and methods}

\section{Participants}

Participants in this study were 60 young adults (18 males) ages 18 to 23 years ( $M=19.05$ years, $S D=1.05$ years) and
58 community-dwelling older adults ( 18 males) ages 65 to 81 years ( $M=71.17$ years, $S D=4.14$ years). Participants who required glasses or corrective lenses wore them on the day of testing, and no participants reported using hearing aids. All participants were native English speakers and completed the Shipley vocabulary test (Shipley, 1940). Young adults in our study had significantly poorer vocabulary knowledge $(M=$ $30.83, S D=3.61)$ than did older adults $(M=34.98, S D=$ $2.98), t(112.80)=-6.79, p<.001$.

\section{Stimuli and materials}

Stimuli in the hearing task were 84 high-predictability sentences (hereafter referred to as congruent sentences; e.g., She put the toys in the box) and 42 carrier sentences (hereafter referred to as baseline sentences; e.g., The word is page) selected from the Revised Speech Perception in Noise Test (Bilger, Nuetzel, Rabinowitz, \& Rzeczkowski, 1984) or created by the researchers. For each congruent sentence, an incongruent sentence was created by changing either the first or last phoneme in the sentence-final target word to form an alternative word that was not predicted by the sentence context (e.g., She put the toys in the fox). The length, frequency, and phonological neighborhood density of all target words were gathered from the English Lexicon Project (Balota et al., 2007). The length of target words in the congruent ( $M$ $=4.35, S D=.99)$, incongruent $(M=4.36, S D=1.05)$, and baseline conditions $(M=4.08, S D=.73)$ did not differ significantly, $F(2,197)=1.35, p>.05$. The frequency of target words in the congruent $(M=53,839 ; S D=120,601.25$, incongruent $(M=57,573 ; S D=86,165.11)$, and baseline conditions $(M=59,267 ; S D=115,873.36)$ also did not differ significantly, $F(2,197)=.04, p>.05$. Finally, the phonological neighborhood density of target words in the congruent $(M=19.22$, $S D=11.03)$, incongruent $(M=19.20, S D=11.45)$, and baseline conditions $(M=18.95, S D=9.73)$ did not differ significantly, $F(2,197)=.01, p>.05$. All sentences were recorded at $44,100 \mathrm{~Hz}$ and 16-bit resolution. Sentences were recorded in a double-walled, sound-attenuating booth, and were spoken at a normal rate by a male with a Midwestern American accent. All sentences were played at an average amplitude of $64 \mathrm{~dB}$ sound pressure level (SPL).

\section{Procedure}

Participants were told that they would hear complete sentences with the final word in background noise, and that their task would be to type the word presented in noise. The target word (but not the preceding context) in each sentence was embedded in speech shaped noise that onset $500 \mathrm{~ms}$ prior to the target word and continued for $500 \mathrm{~ms}$ after the offset of the target word. The SNR was set at $-4 \mathrm{~dB}$ SPL for all young adults and at $+1 \mathrm{~dB}$ SPL for all older adults - SNRs found to 
produce approximately $50 \%$ accuracy in the baseline condition from pilot testing. Participants were explicitly warned that the target words would sometimes be predictable based on the context provided by the sentence, but that sometimes the sentence context would be misleading, and were provided examples of congruent and incongruent sentences. After typing the word they heard in the background noise, participants indicated their reason for providing their response by selecting one of three options-hear, know, or guess-based on the remember/know paradigm (Tulving, 1985). Participants were instructed to select "hear" if they heard the specific sounds of the target word when it was presented, so they were certain that the response they typed was the word that was presented. They were told to select "know" if they did not hear the specific sounds of the target word when it was presented, but still knew that their response was the word that was presented. Finally, they were told to select "guess" if they were purely guessing with no idea of the correct answer. The descriptions for each of these response types were presented on screen each time participants made the hear/know/guess judgement. There was no time limit for identification of the target word or for the hear/know/guess judgement.

Participants completed six practice trials, consisting of two baseline, two congruent, and two incongruent sentences, followed by 120 test trials consisting of 40 baseline, 40 congruent, and 40 incongruent sentences. It should be noted that the sentence context was only an accurate cue for responding in 40 out of 120 total trials (33.33\%), and therefore, if anything, participants should be biased against using the sentence context as a basis for responding. In order to reduce error variance from differences in presentation order, sentences within the practice and test blocks were randomized once, and this same order was used for all participants. Each highpredictability sentence was presented with either the congruent or incongruent target word, and this was counterbalanced across participants so that congruent and incongruent versions of the same sentence were never presented to the same participant. A 1,000-ms pure tone was presented to signal the start of each new trial.

\section{Statistical analyses}

We conducted mixed-effects logistic regression analyses using the glmer function in the lme4 package in R (Bates, Maechler, Bolker, \& Walker, 2015) to ensure that the context manipulation had the predicted effects on accuracy. Level 1 of the model included an intercept term corresponding to the odds of an accurate response in the baseline condition and two dummy-coded variables corresponding to the change in performance from the baseline condition to the congruent and incongruent context conditions, each of which was allowed to vary randomly across participants. At Level 2 of the model, we included a dummy-coded group variable (young adults coded 0 , older adults coded 1 ), and the interaction of the group variable with the congruent and incongruent dummy variables from Level 1. This allowed us to assess the change in the odds of an accurate response from the young adult group to the older adult group in the baseline condition, and the difference in the magnitude of change between young and older adults from the baseline condition to the congruent and incongruent conditions. The dependent variable was trial-by-trial accuracy. Fixed-effect estimates were converted to odds ratios $(O R)$ in the presented tables to facilitate interpretation.

Susceptibility to false hearing was assessed using a second mixed-effects logistic regression model. This model included only an intercept and group, allowing us to assess differences in susceptibility to false hearing across groups. We then conducted a mixed-effects multinomial regression model using the brm function from the brms package in R (Bürkner, 2017) to assess the relative odds of judging a false hearing experience as heard relative to known or guessed. The data used in this model were a subset of data that included only cases of false hearing in the incongruent condition. The model included an intercept term and group as predictors, and the intercept term was allowed to vary randomly across subjects. False hearing responses classified as heard were used as the reference condition, allowing us to assess the relative odds of classifying a response as heard relative to known or guessed, and whether this differed across age groups. Fixed-effect estimates (output as logits) were exponentiated to yield an odds ratio to facilitate interpretation. Since the brms package does not calculate $p$ values, we will report the $95 \%$ confidence intervals $(\mathrm{CI})$ around the exponentiated fixed-effect estimates. A $95 \%$ CI that does not include 1.00 can be interpreted as a significant effect at the $\alpha=.05$ level.

\section{Results}

\section{Accuracy}

The proportion of accurate responses in each condition of the hearing task are presented in Fig. 1. Trends in the hearing task are consistent with those of Rogers et al. (2012, Experiment 2) and Sommers et al. (2015, Experiment 2). While participants achieved accuracy between $50 \%$ and $70 \%$ on average in the baseline condition, they demonstrated nearly perfect performance when the target word was preceded by a congruent semantic context. On the other hand, performance suffered when the target word was preceded by an incongruent semantic context. The mixed-effects logistic regression model confirmed these trends (see Table 1). First, it is important to note that the groups differed in the baseline condition, with the odds of an older adult accurately identifying the target word being 1.35 times greater than young adults $(z=1.35, p<.05)$. This was surprising given that we used SNRs identified in a pilot study to equate the age groups in baseline performance. 


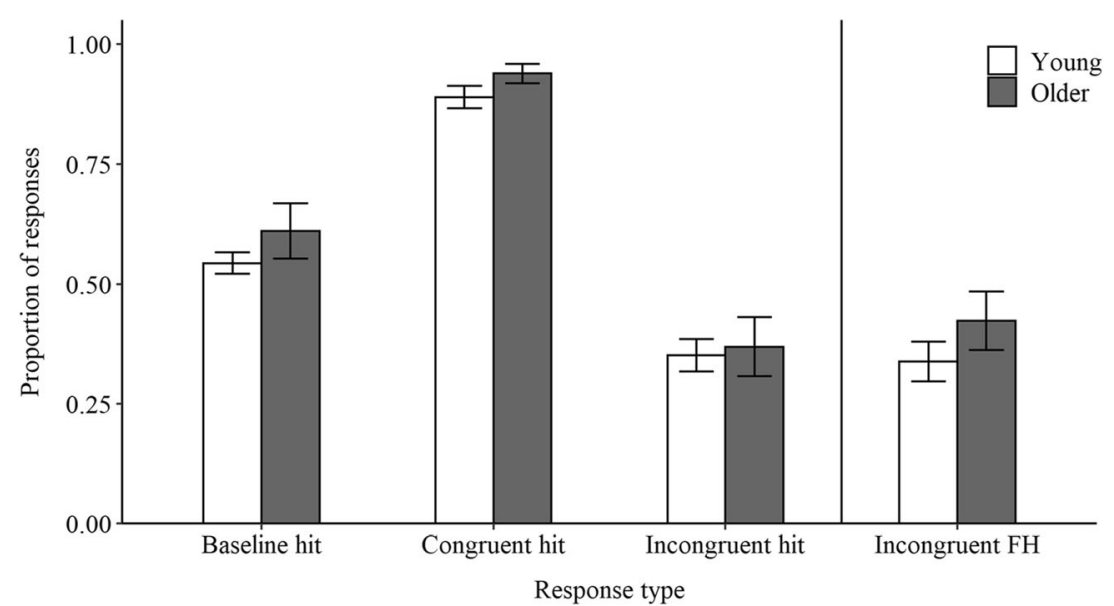

Fig. 1 Mean proportion of correct responses (hits) across possible response types and cases of false hearing (FH) in the hearing task. Error bars represent $95 \%$ confidence intervals

One potential consequence of the inequality between groups in the baseline condition is that older adults may have a more limited ability to benefit from congruent context due to their elevated baseline performance. However, our data suggest that this was not the case. Whereas we found that the odds of correctly identifying the target word was more than 8.34 times greater in the congruent condition than in the baseline condition for young adults $(z=16.59, p<.001)$, older adults

Table 1 Odds of an accurate response by condition in the hearing task

\begin{tabular}{llll}
\hline Predictors & \multicolumn{4}{l}{ Accurate response } \\
\cline { 2 - 4 } & Odds ratios & CI & $p$ \\
\hline (Intercept) & 1.20 & $1.00,1.44$ & .049 \\
Congruent & 8.34 & $6.49,10.72$ & $<.001$ \\
Incongruent & 0.43 & $0.34,0.53$ & $<.001$ \\
Group & 1.35 & $1.04,1.75$ & .026 \\
Congruent:Group & 1.76 & $1.20,2.59$ & .004 \\
Incongruent:Group & 0.70 & $0.51,0.95$ & .023 \\
Random effects & & & \\
$\sigma^{2}$ & 3.29 & & \\
$\tau_{00}$ Subject & 0.42 & & \\
$\tau_{11 \text { Subject.Congruent }}$ & 0.53 & & \\
$\tau_{11 \text { Subject.Incongruent }}$ & 0.47 & & \\
$\rho_{01}$ & -0.13 & & \\
& -0.02 & & \\
ICC & 0.18 & & \\
$N$ Subject & 118 & & \\
Observations & 14,160 & & \\
Marginal $R^{2} /$ Conditional $R^{2}$ & $0.348 / 0.463$ & & \\
\hline
\end{tabular}

Note. $\mathrm{CI}=95 \%$ confidence interval around the odds; $\sigma^{2}=$ residual variance in model; $\tau_{11}=$ variation in odds of a correct response within a given condition across subjects; $\rho_{01}=$ correlation of deviations from the average odds between the baseline condition and the specified condition across subjects; ICC $=$ intraclass correlation coefficient, a measure of response consistency within subjects actually experienced significantly greater benefit from congruent context than did young adults $(O R=1.76, z=2.90, p$ $<.01)$. Similarly, whereas the odds of correctly identifying the target word in the incongruent condition for young adults was 2.35 times worse than in the baseline condition $(z=-7.93, p<$ $.001)$, older adults experienced an even greater detriment to performance $(O R=.70, z=-2.28, p<.05)$. Thus, despite older adults being more likely than young adults to accurately identify the target word in the baseline condition, older adults were influenced by available contextual cues to a greater degree than were young adults.

\section{False hearing}

We next investigated group differences in susceptibility to false hearing in incongruent trials of the hearing task. We found that both young and older adults frequently reported

Table 2. Odds of false hearing on incongruent trials in the hearing task

\begin{tabular}{llll}
\hline Predictors & \multicolumn{2}{l}{ False hearing } & \\
\cline { 2 - 4 } & Odds ratios & CI & $p$ \\
\hline (Intercept) & 0.49 & $0.38,0.62$ & $<.001$ \\
Group & 1.41 & $1.00,2.00$ & .051 \\
Random effects & & & \\
$\sigma^{2}$ & 3.29 & & \\
$\tau_{00 \text { Subject }}$ & 0.80 & & \\
ICC & 0.20 & & \\
$N_{\text {Subject }}$ & 118 & & \\
Observations & 4,720 & & \\
Marginal $R^{2} /$ Conditional $R^{2}$ & $0.007 / 0.202$ & & \\
\end{tabular}

Note. $\mathrm{CI}=95 \%$ confidence interval around the odds; $\sigma^{2}=$ residual variance in model; $\tau_{00}=$ variation in odds of false hearing in the incongruent condition across subjects; ICC $=$ intraclass correlation coefficient, a measure of response consistency within subjects 
hearing the contextually predicted word in the incongruent condition (see Fig. 1). Additionally, as shown in Table 2, the odds that an older adult would experience false hearing was 1.41 times greater than for a young adult, which was significant $(z=1.95, p=.05)$. Thus, we replicated the findings of past studies that older adults are more susceptible to false hearing than are young adults (Rogers et al., 2012; Sommers et al., 2015).

\section{Subjective experience of false hearing}

Finally, we investigated the likelihood of experiencing false hearing rated as heard, known, or guessed across groups. As shown in Fig. 2, both young and older adults frequently (and incorrectly) judged that they had heard the contextually predicted word in the incongruent condition when a similar sounding but unpredicted word was, in fact, presented. Young adults were less likely to judge cases of false hearing as known $(O R=.43,95 \%$ CI $[.32, .58])$ or guessed $(O R=.05,95 \%$ CI $[.03, .08])$ relative to heard. The difference in the odds of judging cases of false hearing as known relative to heard was even greater in older adults $(O R=.47,95 \% \mathrm{CI}[.30, .72])$, but the difference in the odds of judging cases of false hearing as guessed relative to heard did not differ significantly across groups $(O R=$ $1.02,95 \%$ CI $[.54,2.03])$.

\section{Experiment 1 discussion}

Taken together, the results of Experiment 1 look quite similar to those in the studies by Rogers et al. (2012, Experiment 2) and Sommers et al. (2015, Experiment 2). In all cases, accuracy was modulated by the validity of contextual cues, with the highest accuracy when contextual cues were valid

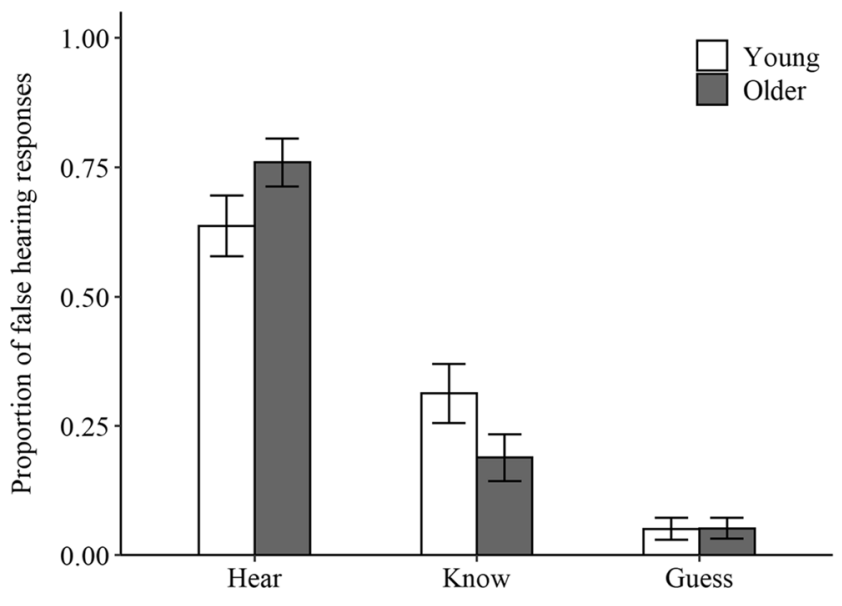

Subjective judgement type

Fig. 2 Proportion of false hearing responses on incongruent trials rated as hear, know, or guess. Error bars represent $95 \%$ confidence intervals predictors of the sentence final word (congruent condition), followed by when there were no contextual cues (baseline condition), and then by when contextual cues were misleading (incongruent condition). Importantly, we also replicated the finding of increased susceptibility to false hearing exhibited by older, relative to young, adults observed in past studies. This finding was particularly remarkable given that older adults exhibited improved audibility of the target word relative to young adults in the baseline condition. Thus, older adults were more likely to use context as a basis for responding than were young adults, even when the auditory stimulus was easier to discern for older adults. This further supports the idea that older adults are overreliant on contextual cues in speech perception, such that they continue to use contextual cues even when they are not valid.

A novel contribution of Experiment 1 was assessing the subjective experience of false hearing through hear/know/ guess judgements. We found that both young and older adults were more likely to judge cases of false hearing as heard as opposed to either known or guessed. Additionally, older adults were more likely to judge cases of false hearing as heard as opposed to known than were young adults. These findings mirror those in false memory, where older adults have been shown to be more likely to judge their false memories as "remembered" than young adults (Jacoby et al., 2005, Experiment 2).

\section{Experiment 2}

Having established robust rates of false hearing in our group of young and older adults, we next sought to determine whether this overreliance on context would transfer to a task in a completely different domain: memory. We employed the same memory task that was used by Jacoby et al. (2005, Experiment 2) described above, which produced high rates of false memory in older adults.

In Experiment 2, we first sought to determine whether memory accuracy was affected by valid, neutral, and misleading contextual cues in a similar way as in the hearing task in Experiment 1. We expected that participants' memory would be best when cued with a valid prime, followed by neutral primes, and then by misleading primes. Based on the findings of Jacoby et al. (2005, Experiment 2), we expected that participants would frequently experience false memories when primes were misleading, and that participants — particularly older adults — would often judge their false memories as remembered. Most importantly, we predicted that older adults who were more susceptible to false hearing in Experiment 1 would be more susceptible to false memory in Experiment 2. This would suggest that false hearing and false memory may share a common 
cognitive mechanism, as opposed to being driven by separate, domain-specific mechanisms.

\section{Materials and methods}

\section{Participants}

Participants were the same 60 young adults and 58 community-dwelling older adults from Experiment 1. Participants completed Experiments 1 and 2 during separate 1-hour sessions on different days.

\section{Stimuli and materials}

Participants completed two phases in this task - a study phase and a test phase. Stimuli in the study phase were 99 semantically related cue-target word pairs (e.g., head-skull). Cue words ranged from three to nine letters in length $(M=5.08$, $S D=1.41)$, and target words ranged from three to seven letters in length $(M=4.59, S D=.79)$. Stimuli in the test phase consisted of the same cues from the study phase matched with a fragment of its paired target created by replacing certain letters with dashes (e.g., head-s-l-). Target words in the test phase had between one and five letters replaced by dashes $(M$ $=2.21, S D=.71)$, and retained between one and four letters $(M=2.38, S D=.63)$, although all but one fragment $(-u$ - for the target word $m u g$ ) retained at least two letters. The primes in the test phase were either the target word from the study phase (congruent condition; e.g., skull), an alternative word that could complete the target fragment and that was semantically related to the cue (incongruent condition; e.g., scalp), or a string of five ampersands ( $\& \& \& \& \&$ ), which provided no predictive value for the fragment completion task (baseline condition). There were no differences in target word length between the congruent $(M=4.57, S D=.73)$, incongruent $(M=$ $4.47, S D=.78)$, and baseline conditions $(M=4.73, S D=.87)$, $F(2,87)=.87, p>.05$. We obtained the $\log$ frequencies for targets in the congruent $(M=9.37, S D=2.01)$, incongruent $(M=9.67, S D=1.55)$, and baseline conditions $(M=9.29, S D$ $=1.74$ ) from the English Lexicon Project (Balota et al., 2007), and found that there were no differences in target-word frequency across conditions, $F(2,87)=.38, p>.05$.

\section{Procedure}

Participants were told to study a list of semantically related word pairs for a later memory test. They were shown six cuetarget word pairs as examples. Following the examples, participants completed the study phase, in which 90 cue-target word pairs were presented. An additional three cue-target word pairs were presented following the study phase to act as a buffer against the recency effect. For young adults, each word pair was presented for 1,000 ms, whereas each word pair was presented for 3,000 ms for older adults, a manipulation used by Jacoby et al. (2005) to equate memory for words in the baseline condition. An interstimulus interval (ISI) of $500 \mathrm{~ms}$ separated each cue-target word pair. All stimuli in the study phase were presented in a predetermined random order, which was used for all participants, and each cuetarget word pair was presented only once.

To equate the interval from the first word in the study phase to the last word in the buffer trials, young adults waited 186 seconds before starting the memory test, whereas older adults started the memory test immediately following the buffer trials. In the memory test, participants were again shown the word pairs from the learning phase, but in each case the second word in the pair was fragmented by substituting dashes for some of the letters. Participants were instructed that their job was to complete the word fragment by typing the word that accurately completed the learned word pair from the study phase. Before each cue-fragment pair was presented, a congruent, incongruent, or baseline prime was flashed on-screen. Participants were explicitly warned at the start of the study that primes would appear, and were told that the prime would either be the target word from the study phase, in which case they should use this word to complete the word fragment, an unstudied word that was semantically related to the presented cue and that could complete the word fragment, in which case they should not use this word to complete the word fragment, or a string of ampersands. The prime remained on screen for $500 \mathrm{~ms}$ and offset $500 \mathrm{~ms}$ before presentation of the cuefragment pair. Participants completed 30 trials in each of the three prime conditions (congruent, baseline, and incongruent), and trials were presented in a predetermined random order. It should be noted that the prime was only an accurate cue for memory in 30 out of 90 total trials (33.33\%), and therefore, if anything, participants should be biased against using the prime as a basis for responding.

After typing the word that completed each cue-target word pair, participants indicated their reason for providing their response by selecting one of three options: remember, know, or guess (Tulving, 1985). Participants were told to select "remember" if they recalled specific details of the word's presentation in the study list, so they were certain that their response was the word from the study list. They were told to select "know" if they knew that they had studied the word they typed, but could not recall specific details about studying it. Finally, they were told to select "guess" if they were purely guessing with no idea of the correct answer. The descriptions for each these response types were presented on screen each time participants made the remember/know/guess judgement.

\section{Statistical analyses}

Statistical analyses were identical to Experiment 1, predicting accurate responses, susceptibility to false memory in general, 
and susceptibility to false memories judged as remembered, known, or guessed. In addition to these analyses, we compared susceptibility to false memory to susceptibility to false hearing from Experiment 1. For this analysis, we created a mixed-effects logistic regression model predicting trial-bytrial false hearing from Experiment 1 . We included each participant's $z$-scored average susceptibility to false memory from Experiment 2 (standardized across all participants) and group as predictors, as well as the interaction between susceptibility to false memory and group. As in the other models conducted in this experiment, the intercept term was allowed to vary randomly across subjects.

\section{Results}

\section{Accuracy}

The proportion of accurate responses in each condition of the memory task are shown in Fig. 3. Our data replicate those of Jacoby et al. (2005), with congruent primes improving memory accuracy relative to baseline primes, and incongruent primes decreasing accuracy relative to baseline primes. These trends were confirmed by our mixed-effects logistic regression model (see Table 3). As in the hearing task in Experiment 1, performance in the age groups was not equated in the baseline condition. The odds of an accurate response in the baseline condition were 1.52 times greater for older adults than young adults $(z=4.05, p<.001)$. The inequality in the baseline condition was surprising given that we used the exact same task as that used by Jacoby et al. (2005, Experiment 2), where young and older adults achieved equivalent performance in the baseline condition. Despite the inequality in the baseline condition, we again observed that the performance of older adult participants was impacted to a greater degree by context than was that of young adults. When the word fragment was preceded by a congruent prime, the odds
Table 3. Odds of an accurate response by condition in the memory task

\begin{tabular}{llll}
\hline \multirow{2}{*}{ Predictors } & \multicolumn{2}{l}{ Accuracy } & \\
\cline { 2 - 4 } & Odds ratios & CI & $p$ \\
\hline Intercept) & 1.22 & $1.06,1.40$ & .006 \\
Congruent & 4.90 & $3.75,6.40$ & $<.001$ \\
Incongruent & 0.52 & $0.41,0.65$ & $<.001$ \\
Group & 1.52 & $1.24,1.87$ & $<.001$ \\
Congruent:Group & 1.63 & $1.09,2.43$ & .018 \\
Incongruent:Group & 0.40 & $0.29,0.56$ & $<.001$ \\
Random effects & & & \\
$\sigma^{2}$ & 3.29 & & \\
$\tau_{00}$ Subject & 0.17 & & \\
$\tau_{11 \text { Subject.Congruent }}$ & 0.69 & & \\
$\tau_{11 \text { Subject.Incongruent }}$ & 0.50 & & \\
$\rho_{01}$ & -0.58 & & \\
& 0.75 & & \\
$N_{\text {Subject }}$ & 118 & & \\
Observations & 10620 & & \\
Marginal $R^{2} /$ Conditional $R^{2}$ & $0.322 / \mathrm{NA}$ & & \\
\hline
\end{tabular}

Note. $\mathrm{CI}=95 \%$ confidence interval around the odds; $\sigma^{2}=$ residual variance in model; $\tau_{11}=$ variation in odds of a correct response within a given condition across subjects; $\rho_{01}=$ correlation of deviations from the average odds between the baseline condition and the specified condition across subjects; ICC = intraclass correlation coefficient, a measure of response consistency within subjects

that young adults would correctly complete the word fragment increased by a factor of 4.90 relative to the baseline condition $(z=11.61, p<.001)$. However, when the prime was misleading (i.e., in the incongruent condition), the odds that young adults would correctly complete the word fragment decreased by a factor of 1.93 relative to the baseline condition $(z=-5.67$, $p<.001)$. The difference between the baseline condition and both the congruent $(O R=1.63, z=2.36, p<.05)$ and incongruent conditions $(O R=.40, z=-5.32, p<.001)$ was greater

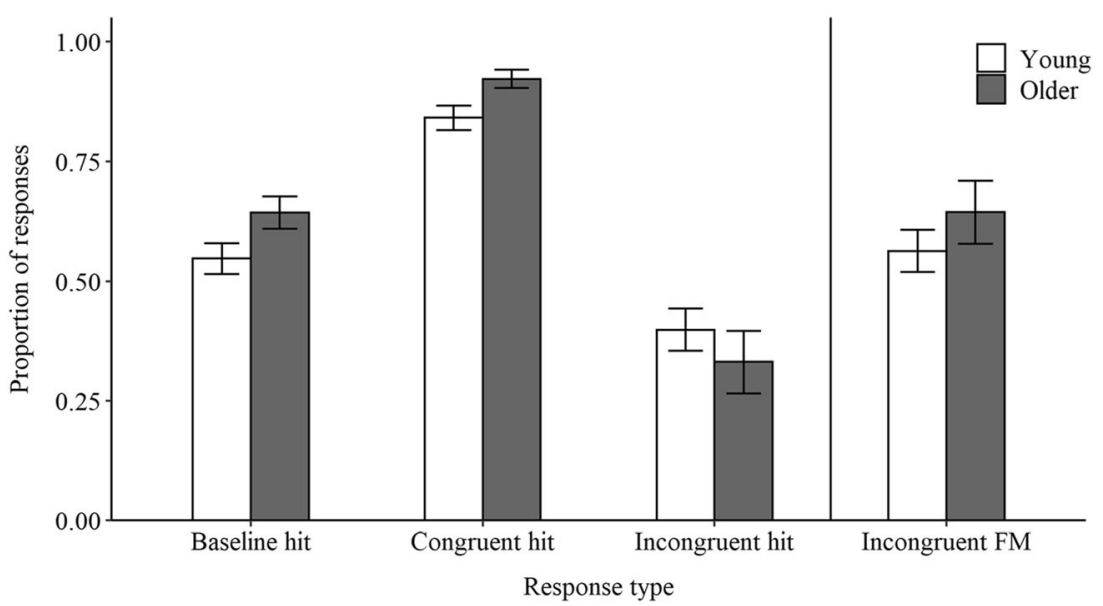

Fig. 3 Mean proportion of correct responses (hits) across possible response types and cases of false memory (FM) in the memory task. Error bars represent $95 \%$ confidence intervals 
in older, relative to young, adults. Thus, as in the hearing task in Experiment 1, accuracy in the memory task was modulated by the validity of available contextual cues, in this case, the primes.

\section{False memory}

We next compared the likelihood of participants incorrectly completing the word fragment with the incongruent prime word (i.e., experiencing a false memory) across age groups (see Table 4). We found that the odds that older adults would experience a false memory was 1.64 times greater than for young adults $(z=2.47, p<.05)$. This replicates the findings of Jacoby et al. (2005, Experiment 2), who reported that older adults were more likely to falsely remember having studied the prime word in the incongruent condition than young adults.

\section{Subjective experience of false memory}

We next investigated the likelihood of experiencing false memories rated as remembered, known, or guessed across groups. As shown in Fig. 4, both young and older adults frequently judged cases of false memory as remembered. However, unlike for false hearing in Experiment 1, young adults were not less likely to judge cases of false memory as either known $(O R=1.17,95 \%$ CI [ .73, 1.86]) or guessed $(O R$ $=1.36,95 \% \mathrm{CI}[.85,2.21])$ relative to remembered. The difference in the odds of judging cases of false memory as known relative to remembered was not different in older adults than in young adults $(O R=.52,95 \%$ CI $[.27,1.00])$. However, the difference in the odds of judging cases of false memory as guessed relative to remembered was greater in older adults $(O R=.19,95 \%$ CI $[.09, .37])$. In fact, whereas young adults

Table 4. Odds of false memory across age groups

\begin{tabular}{llll}
\hline \multirow{2}{*}{ Predictors } & \multicolumn{2}{l}{ False memory } & \\
\cline { 2 - 4 } & Odds ratios & CI & $p$ \\
\hline (Intercept) & 1.32 & $1.01,1.74$ & .044 \\
Group & 1.64 & $1.11,2.43$ & .013 \\
Random effects & & & \\
$\sigma^{2}$ & 3.29 & & \\
$\tau_{00 \text { Subject }}$ & 1.00 & & \\
ICC & 0.23 & & \\
$N_{\text {Subject }}$ & 118 & \\
Observations & 3540 & \\
Marginal $R^{2} /$ Conditional $R^{2}$ & $0.014 / 0.244$ & & \\
\hline
\end{tabular}

Note. CI $=95 \%$ confidence interval around the odds; $\sigma^{2}=$ residual variance in model; $\tau_{00}=$ variation in odds of false memory in the incongruent condition across subjects; ICC $=$ intraclass correlation coefficient, a measure of response consistency within subjects

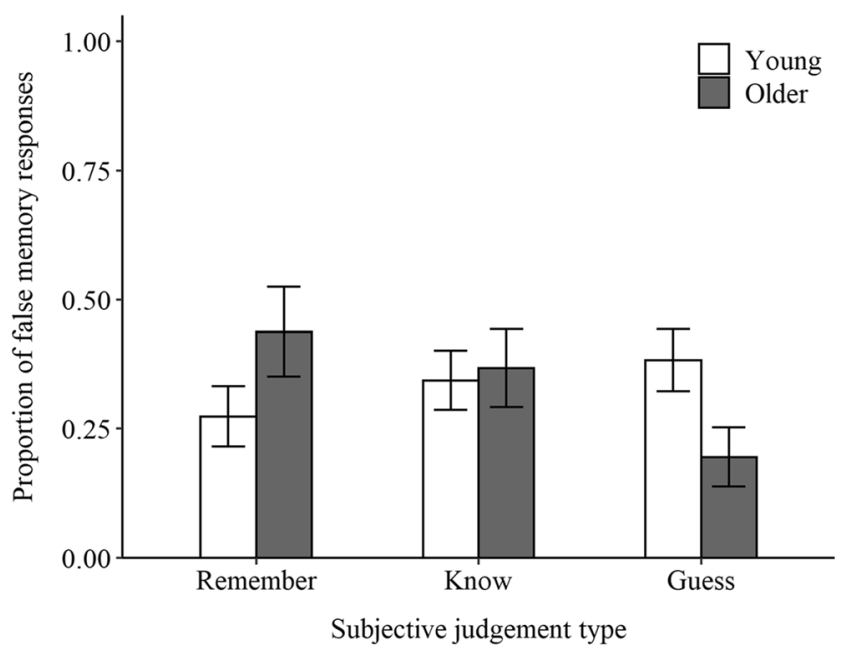

Fig. 4 Proportion of false memory responses on incongruent trials rated as remember, know, or guess. Error bars represent 95\% confidence intervals

were nonsignificantly more likely to judge their false memories as guessed than remembered, older adults displayed the opposite pattern.

\section{Relationship between false memory and false hearing}

Our final set of analyses sought to answer the question of whether performance on the memory task could be used to predict performance on the hearing task from Experiment 1. We first conducted a basic Pearson's product moment correlation between average susceptibility to false hearing and average susceptibility to false memory on incongruent trials of the hearing and memory tasks, respectively (see Fig. 5). The correlation was significant $(r=.39, p<.001)$, indicating that participants who were more susceptible to false hearing also tended to be more susceptible to false memory.

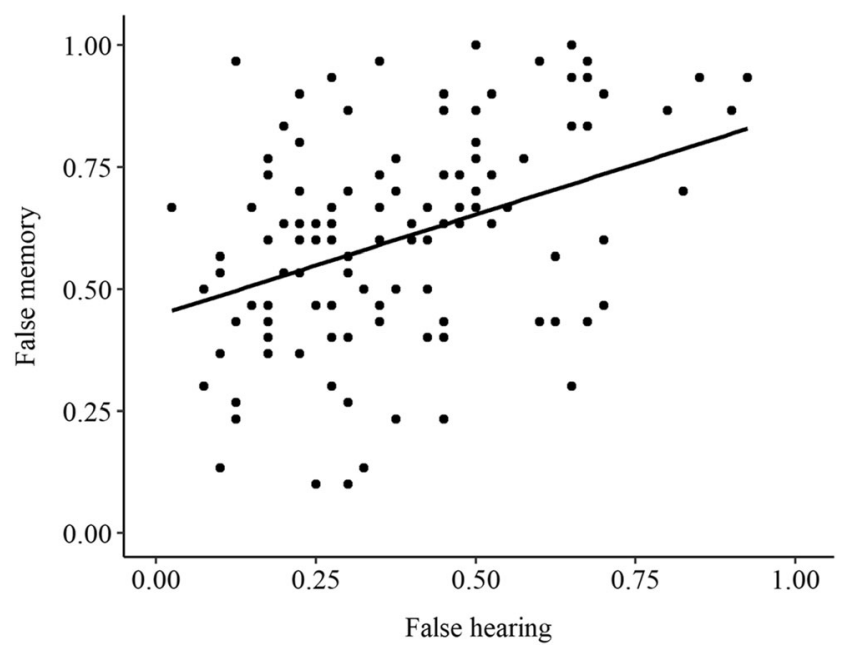

Fig. 5 Correlation between average susceptibility to false hearing and average susceptibility to false memory $(r=.39)$ 
The results of the mixed-effects logistic regression model supported the same relationship. We found that the interaction between susceptibility to false memory and group was nonsignificant $(O R=1.20, z=.99, p>.05)$, so we removed it from the model since it may obscure the main effects of false memory and group. In the updated model (see Table 5), a one standard deviation increase in average susceptibility to false memory increased the odds of experiencing false hearing by a factor of 1.45, which was significant $(z=4.34, p<.001)$. Thus, participants who were more susceptible to false memory also tended to be more susceptible to false hearing, and this relationship did not differ across age groups.

\section{Experiment 2 discussion}

In all, the results of Experiment 2 replicated those of Jacoby et al. (2005, Experiment 2), showing that both young and older adults employ available contextual cues as a basis for responding in the memory task. Additionally, as in the hearing task in Experiment 1, older adults - and, to a lesser degree, young adults - continued to use contextual cues even when context was misleading, resulting in high rates of false memory. Thus, participants-older adults in particular-were overly reliant on contextual cues in both hearing and memory.

Similarly to false hearing in Experiment 1, older adults were more likely to judge their false memories as remembered relative to known or guessed. Unlike in Experiment 1, however, young adults' subjective experience was better tuned to the accuracy of their response, as they were nonsignificantly more likely to judge false memories as guessed as opposed to remembered or known. This suggests that young adults had

Table 5. The relationship between susceptibility to false memory and susceptibility to false hearing

\begin{tabular}{llll}
\hline Predictors & \multicolumn{2}{l}{ False hearing } & \\
\cline { 2 - 4 } & Odds ratios & CI & $p$ \\
\hline (Intercept) & 0.52 & $0.42,0.66$ & $<.001$ \\
Group & 1.23 & $0.89,1.71$ & .212 \\
Average false memory $(z$ scored $)$ & 1.45 & $1.22,1.71$ & $<.001$ \\
Random effects & & & \\
$\sigma^{2}$ & 3.29 & & \\
$\tau_{00}$ Subject & 0.67 & & \\
ICC & 0.17 & & \\
$N_{\text {Subject }}$ & 118 & & \\
Observations & 4720 & & \\
Marginal $R^{2} /$ Conditional $R^{2}$ & $0.039 / 0.202$ & & \\
\hline
\end{tabular}

Note. $\mathrm{CI}=95 \%$ confidence interval around the odds; $\sigma^{2}=$ residual variance in model; $\tau_{00}=$ variation in odds of false hearing in the incongruent condition across subjects; ICC $=$ intraclass correlation coefficient, a measure of response consistency within subjects better metacognition regarding the accuracy of their false memory responses than their false hearing responses.

Importantly, older adults' susceptibility to false memory in Experiment 2 was predictive of their susceptibility to false hearing in Experiment 1. Participants who were more susceptible to false hearing in Experiment 1 also tended to be more susceptible to false memory in Experiment 2. These findings suggest that the ability to resist misleading contextual cues in speech perception and in memory may rely, at least in part, on a common cognitive mechanism as opposed to separate, domain-specific mechanisms.

It is notable that there was no significant age difference in the magnitude of the relationship between false hearing and false memory, as indicated by the nonsignificant interaction between age group and average susceptibility to false memory predicting trial-by-trial false hearing. One explanation for this lack of age differences is that our study may simply have been underpowered to detect age-group differences in the magnitude of the relationship between false hearing and false memory. Indeed, looking at the raw correlations between false hearing and false memory revealed a larger correlation in older adults $(r=.44, p<.001)$ than in young adults $(r=.21$, $p=.11)$. This suggests that the relationship between false hearing and false memory may have been greater in older, relative to young, adults. Additionally, age differences in the magnitude of the relationship between false hearing and false memory may have been mitigated by unequal performance between groups in the baseline condition of both the hearing and memory tasks. Since older adults were better able to identify the target word in the absence of contextual cues than were young adults, they may have had less need to rely on context as a basis for responding relative to if their performance in the baseline condition had been equal to that of the young adult group. This would diminish the positive relationship between age and reliance on context as a basis for responding reported in past studies (Rogers et al., 2012; Sommers et al., 2015), which could, in turn, mitigate age differences in the magnitude of the correlation between false hearing and false memory. Future studies should test whether age groups differ in the magnitude of the relationship between false hearing and false memory with a larger sample size and with age groups equated in baseline performance to determine whether this effect emerges.

\section{General discussion}

In the present study, we compared young and older adults' performance on a hearing task and a memory task to determine whether susceptibility to false hearing was correlated with susceptibility to false memory. We were able to successfully replicate the findings of Sommers et al. (2015, Experiment 2) and Jacoby et al. (2005, Experiment 2) using 
their respective hearing and memory tasks. In both tasks, adding valid predictive context significantly improved performance relative to when no context was present, supporting the findings of past studies showing that young and older adults are able to use available contextual cues to support memory (Jacoby, 1999; Jacoby et al., 2005, Experiment 2) and hearing (Dubno, Ahlstrom, Horwitz, 2000; Hutchinson, 1989; Rogers et al., 2012, Experiment 2; Sommers et al., 2015, Experiment 2). However, introducing misleading context was detrimental to performance relative to baseline, with participants often providing the contextually predicted response, leading to robust rates of both false hearing and false memory. Importantly, we found that participants who were more prone to false hearing also tended to be more prone to false memory. This finding supports the idea that false hearing and false memory share at least one common underlying mechanism.

As noted above, the process dissociation framework (Jacoby, 1991) provides a platform for understanding how the distinct tasks used to elicit false hearing and false memories may engage similar underlying mechanisms. Specifically, the process dissociation framework provides a method for establishing the independent contributions of controlled and automatic processes to memory and speech perception. Our findings suggest that susceptibility to both false hearing and false memory stems primarily from an overreliance on automatic processes, resulting in an inability to resist a prepotent but inaccurate response. On incongruent trials of the hearing task, participants had to resist a response option that was made highly salient by the preceding sentence context and listen closely to the presented target word in order to give the unpredicted (but correct) response. Similarly, on incongruent trials of the memory task, an unstudied word that could complete the fragment was presented as a prime before the fragment completion task, making it a highly salient response option. To correctly complete the fragment, participants had to resist the salient but false prime and engage in conscious recollection to remember the studied word that completed the word pair. Therefore, a failure to resist a salient, automatic response is one possible mechanism to explain the correlation between false hearing and false memory observed in our study.

One model that has been used to explain age-related increases in automatic processes as a basis for responding is the capture model (Jacoby et al., 2005; see also Millar, Balota, Bishara, \& Jacoby, 2018). According to this model, false memories can arise from two different processes. First, participants may be "captured" by the available contextual cues, leading them to provide the context-based response without attempting to recollect the studied target word. Second, false memories can occur when the individual is not captured by context, but their attempt at recollecting the studied target word fails. In this second scenario, the individual may provide the contextually predicted response because it is an easily accessible and plausible response option. Jacoby et al. found that the capture model — which included parameters for capture, recollection, accessibility bias (i.e., the probability of generating the prime in the memory task when attempts at recollection failed), and word generation (i.e., the probability of generating the preselected alternative word in the memory task) - provided a better fit to their data than a model that only included parameters for recollection, accessibility bias, and word generation. This suggests that automatic capture by the prime made an important contribution to responding in their memory task. This was especially true for older adults in their sample, who were more prone to capture than were young adults. In fact, the authors found that young and older adults differed only in the capture parameter, and did not differ in recollection, accessibility bias, or word generation. More recently, however, Millar et al. (2018) found that age differences in the same memory task were best explained by a model that accounted for both older adults' increased susceptibility to capture and decreased likelihood of recollecting the target word relative to young adults, with no age differences in accessibility bias or word generation.

Importantly, the recollection parameter in the studies by Jacoby et al. (2005, Experiment 2) and Millar et al. (2018) reported above corresponded to performance in the baseline condition of the memory task: Answering correctly in the baseline condition required participants to remember the target word without the influence of contextual cues. As was true of the recollection parameter, age groups did not differ in the baseline condition of the study by Jacoby et al., whereas older adults displayed poorer performance than did young adults in the baseline condition of the study by Millar et al. In the present study, it is interesting to note that older adults were more likely to experience false hearing and false memory than were young adults despite having higher baseline accuracy in both tasks (i.e., being better able to hear and recollect the target words). This lends further support to the idea that agerelated declines in cognitive control - as opposed to declines in hearing or memory per se - gave rise to older adults' increased susceptibility to false hearing and false memory in the present study.

Although the capture model has not been tested with regard to false hearing, the same parameters that were used successfully to explain false memory can also be applied to the false hearing paradigm. When the preceding sentence context is highly predictive of a sentence-final word in noise, individuals may be captured by the context, leading participants to report hearing the word predicted by context without attending to the target word itself. As was the case with false memory, false hearing can also occur when individuals are unable to identify the target word (in this case because of the presence of noise), leading them to provide the contextually predicted response due to its accessibility and plausibility. The fit of the capture 
model to false hearing should be tested in future studies, as it may help to dissociate between automatic capture by context and context-based guessing, and may provide a framework for understanding older adults' increased susceptibility to false hearing relative to young adults. Additionally, fitting the capture model to hearing data may help to distinguish between age-related deficits in cognitive control and sensation, just as it has been useful for distinguishing between age-related deficits in cognitive control and memory per se (Jacoby et al., 2005; Millar et al., 2018). Finding that age-related deficits in cognitive control contribute to errors in hearing would motivate development of cognitive interventions to supplement sensory interventions (e.g., hearing aids) when treating hearing loss, which could improve speech perception outcomes for older adults.

The idea that false memory and false hearing can occur when the individual is captured by available contextual cues is similar to a prevalent theory that false memories result, in part, from failures to inhibit a prepotent response (see Balota et al., 1999). According to this theory, contextually predicted but unpresented words or events can become activated due to their association with actually presented words or contextual cues, which can lead participants to falsely remember that the unpresented word or event had been present in the studied material. Within this framework, inhibitory control is thought to be required to suppress activation of words or events that become activated due to spreading activation rather than from their presence in the studied information. Since inhibitory control is thought to be needed to suppress a prepotent response, it is possible that poor inhibitory control increases the likelihood of being captured by misleading contextual cues. Thus, individual differences in inhibitory control could account for differences in susceptibility to both false memory and false hearing. Differences in inhibitory control could also account for older adults' greater susceptibility to both false memory (Jacoby et al., 2005) and false hearing (Rogers et al., 2012; Sommers et al., 2015) relative to young adults, as older adults have been found to exhibit poorer inhibitory control than young adults (Cohn, Dustman, \& Bradford, 1984; Hasher, Quig, \& May, 1997; Hasher \& Zacks, 1988; Sommers $\&$ Huff, 2003).

In support of the role of inhibitory control in false memory, two studies (Colombel, Tessoulin, Gilet, \& Corson, 2016; Sommers \& Huff, 2003) have shown that poor inhibitory control is related to increased susceptibility to associative false memories in the Deese-Roediger-McDermott (DRM) paradigm (Deese, 1959; Roediger \& McDermott, 1995). Sommers and Huff (2003, Experiment 2), for example, showed that poor inhibitory control - as measured by an auditory Stroop task - was related to increased susceptibility to both false recall and false recognition in a DRM paradigm using phonologically related word lists. Additionally, several studies have found that age-related differences in executive functioning - a set of cognitive processes including inhibitory control that regulate planning and goal-directed behavior (see Wheeler, Stuss, \& Tulving, 1997) - accounts for age-differences in susceptibility to false memory, such that age-differences in false memory are reduced or disappear when comparing older adults with high executive functioning to young adults (Butler, McDaniel, Dornburg, Price, \& Roediger, 2004; McCabe, Roediger, McDaniel, \& Balota, 2009; Roediger \& Geraci, 2007).

The idea that both false hearing and false memory may result from failures of inhibitory control can help to explain why participants often reported the subjective experience of "hearing" or "remembering" in cases of false hearing and false memory in our study. If it is the case that false hearing and false memory can occur when a response option becomes highly activated due to preceding context, it is plausible that the subjective experience in these cases may be similar to that of veridical hearing or memory. In our hearing task, there are two sources of information upon which to base a response: context and the sensory signal. If one's perceptual experience is determined by whatever response gains the most activation, as would be argued by models of speech perception, such as the neighborhood activation model (Luce \& Pisoni, 1998), then the source of the activation-context or sensory information - should not matter when it comes to the perceptual experience. Similarly, in memory, one's subjective memory can be determined by the degree of activation from context relative to that of the original memory trace. Thus, in cases where participants reported the experience of hearing or remembering in cases of false hearing and false memory, it may be the case that the activation provided by context exceeded that of the sensory signal or memory trace.

It is clear from our data, however, that cases of false hearing and false memory are not always accompanied by the subjective experience of hearing or remembering. In the case of false memory in particular, participants frequently characterized their responses as either known or guessed. This would be expected based on the explanation of false memory provided by the capture model proposed by Jacoby et al. (2005). As described above, false memory-and, we argue, false hearing - can result either from being captured by context or from context-based guessing. Jacoby et al. argued that whereas cases of false memory resulting from capture would be characterized as remembered or known, cases of false memory that do not result from capture would be more likely to be characterized as guesses.

Based upon this explanation, both false hearing and false memory in our study were more likely to result from capture than context-based guessing, evidenced by the proportion of responses characterized as heard/remembered or known relative to guessed. However, our findings suggest that a greater proportion of cases of false hearing (see Fig. 2) resulted from capture than false memory (see Fig. 4). This was likely due to 
differences in the strength of contextual cues in the hearing and memory tasks. In the hearing task, context was provided by highly predictive sentences, whereas in the memory task, context was a single prime word. Therefore, we suggest that the likelihood of being captured by context is determined in part by the predictive strength of available contextual cues. Future studies should investigate the relationship between predictive strength and subjective experience in false hearing and false memory.

\section{Conclusions}

Previous studies (Jacoby, Rogers, Bishara, \& Shimizu, 2012; Rogers et al., 2012) have suggested that false hearing and false memory result from a common mechanism. The present study is the first to explore this hypothesis directly by comparing rates of false hearing to those of false memory in young and older adults. We found that participants who were more prone to false hearing were also more prone to false memory, supporting the idea that the two phenomena share at least one common mechanism. As in past studies (Jacoby et al., 2012; Rogers et al., 2012), we suggested that the mechanism underlying both false hearing and false memory is a deficit in inhibitory control, leading participants to respond based on available context, even when the context is misleading. In support of this hypothesis, we drew upon the capture model proposed by Jacoby et al. (2005) and described its compatibility with the patterns observed in our study, both in terms of response accuracy and subjective experience.

Acknowledgements We would like to acknowledge the hard work of the research assistants in the Speech \& Hearing lab at Washington University in St. Louis-Adam Isaacs, Aryn Lyke, Audrey Ulfers, Davis Holmes, Forrest Whiting, and Zi'Onay Walker-who aided in data collection.

Open practices statement The data and materials for all tasks in this experiment are available (https://osf.io/dtvxy/?view only= $258 \mathrm{f} 71 \mathrm{~d} 332 \mathrm{c} 148 \mathrm{ed} 9741 \mathrm{~d} 3 \mathrm{ea} 66 \mathrm{fb} 4 \mathrm{bc} 1)$. This experiment was not preregistered.

Funding This project was funded by the National Institutes of Health (NIH) [1 R21AG041958-01A1].

\section{References}

Alba, J. W., \& Hasher, L. (1983). Is memory schematic? Psychological Bulletin, 93, 203-231. doi:https://doi.org/10.1037/0033-2909.93.2. 203

Balota, D. A., Cortese, M. J., Duchek, J. M., Adams, D., Roediger, H. L., III, McDermott, K. B., \& Yerys, B. E. (1999). Veridical and false memories in healthy older adults and in dementia of the Alzheimer's type. Cognitive Neuropsychology, 16, 361-384. doi:https://doi.org/ 10.1080/026432999380834

Balota, D. A., Yap, M. J., Cortese, M. J., Hutchison, K. A., Kessler, B., Loftis, B., Neely, J. H., Nelson, D. L., Simpson, G. B., \& Treiman,
R. (2007). The English Lexicon Project. Behavior Research Methods, 39, 445-459. doi:https://doi.org/10.3758/BF03193014

Bartlett, F. C. (1932). Remembering: A study in experimental and social psychology. Cambridge, England: Cambridge University Press.

Bates, D., Maechler, M., Bolker, B., \& Walker, S. (2015). Fitting linear mixed-effects models using lme4. Journal of Statistical Software, 67, 1-48. doi:https://doi.org/10.18637/jss.v067.i01

Bilger, R. C., Nuetzel, J. M., Rabinowitz, W. M., \& Rzeczkowski, C. (1984). Standardization of a test of speech perception in noise. Journal of Speech and Hearing Research, 27, 32-48. doi:https:// doi.org/10.1121/1.2017541

Bugelski, B. R., \& Alampay, D. A. (1961). The role of frequency in developing perceptual sets. Canadian Journal of Psychology, 15, 205-211. doi:https://doi.org/10.1037/h0083443

Bürkner, P. (2017). brms: An R package for Bayesian multilevel models using Stan. Journal of Statistical Software, 80, 1-28. doi:https://doi. org/10.18637/jss.v080.i01

Butler, K. M., McDaniel, M. A., Dornburg, C. C., Price, A. L., \& Roediger, H. L., III (2004). Age differences in veridical and false recall are not inevitable: The role of frontal lobe function. Psychonomic Bulletin \& Review, 11, 921-925. doi:https://doi.org/ 10.3758/BF03196722

Cohn, N. B., Dustman, R. E., \& Bradford, D. C. (1984). Age-related decrements in Stroop color test performance. Journal of Clinical Psychology, 40, 1244-1250. doi:https://doi.org/10.1002/10974679(198409)40:5<1244::AID-JCLP2270400521>3.0.CO;2-D

Colombel, F., Tessoulin, M., Gilet, A.-L., \& Corson, Y. (2016). False memories and normal aging: Links between inhibitory capacities and monitoring processes. Psychology and Aging, 31, 239-248. doi:https://doi.org/10.1037/pag0000086

Coren, S., \& Girgus, J. S. (1978). Seeing is deceiving: The psychology of visual illusions. Hillsdale, NJ: Erlbaum.

Deese, J. (1959). On the prediction of occurrence of particular verbal intrusions in immediate recall. Journal of Experimental Psychology, 58, 17-22. doi:https://doi.org/10.1037/h0046671

Dubno, J. R., Ahlstrom, J. B., \& Horwitz, A. R. (2000). Use of context by young and aged adults with normal hearing. Journal of the Acoustical Society of America, 107, 538-546. doi:https://doi.org/ $10.1121 / 1.428322$

Hasher, L., \& Zacks, R. T. (1988). Working memory, comprehension, and aging: A review and a new view. Psychology of Learning and Motivation, 22, 193-225. doi:https://doi.org/10.1016/S00797421(08)60041-9

Hasher, L., Quig, M. B., \& May, C. P. (1997). Inhibitory control over nolonger relevant information: Adult age differences. Memory \& Cognition, 25, 286-295. doi:https://doi.org/10.3758/BF03211284

Hay, J. F., \& Jacoby, L. L. (1999). Separating habit and recollection in young and older adults: Effects of elaborative processing and distinctiveness. Psychology and Aging, 14, 122-134. doi:https://doi. org/10.1037/0882-7974.14.1.122

Hutchinson, K. M. (1989). Influence of sentence context on speech perception in young and older adults. Journal of Gerontology: Psychological Sciences, 44, 36-44. doi:https://doi.org/10.1093/ geronj/44.2.p36

Jacoby, L. L. (1991). A process dissociation framework: Separating automatic from intentional uses of memory. Journal of Memory and Language, 30, 513-541. doi:https://doi.org/10.1016/0749596X(91)90025-F

Jacoby, L. L. (1999). Deceiving the elderly: Effects of accessibility bias in cued-recall performance. Cognitive Neuropsychology, 16, 417-436. https://doi.org/10.1080/026432999380861

Jacoby, L. L., \& Rhodes, M. G. (2006). False remembering in the aged. Current Directions in Psychological Science, 15, 49-53. doi:https:// doi.org/10.1111/j.0963-7214.2006.00405.x

Jacoby, L. L., Bishara, A. J., Hessels, S., \& Toth, J. P. (2005). Aging, subjective experience, and cognitive control: Dramatic false 
remembering by older adults. Journal of Experimental Psychology: General, 134, 131-148. doi:https://doi.org/10.1037/0096-3445.134. 2.131

Jacoby, L. L., Rogers, C. S., Bishara, A. J., \& Shimizu, Y. (2012). Mistaking the recent past for the present: False seeing by older adults. Psychology and Aging, 27, 22-32. doi:https://doi.org/10. 1037/a0025924

Jennings, J. M., \& Jacoby, L. L. (1997). An opposition procedure for detecting age-related deficits in recollection: Telling effects of repetition. Psychology and Aging, 12, 352-361. doi:https://doi.org/10. 1037/0882-7974.12.2.352

Luce, P. A., \& Pisoni, D. B. (1998). Recognizing spoken words: The neighborhood activation model. Ear Hear, 19, 1-36. doi:https:// doi.org/10.1097/00003446-199802000-00001

McCabe, D. P., Roediger, H. L., III, McDaniel, M. A., \& Balota, D. A. (2009). Aging reduces veridical remembering but increases false remembering: Neuropsychological test correlates of rememberknow judgments. Neuropsychologia, 47, 2164-2173. doi:https:// doi.org/10.1016/j.neuropsychologia.2008.11.025

Millar, P. R., Balota, D. A., Bishara, A. J., \& Jacoby, L. L. (2018). Multinomial models reveal deficits of two distinct controlled retrieval processes in aging and very mild Alzheimer disease. Memory \& Cognition, 46, 1058-1075. doi:https://doi.org/10.3758/s13421-0180821-9

Roediger, H. L., III, \& Geraci, L. (2007). Aging and the misinformation effect: A neuropsychological analysis. Journal of Experimental Psychology: Learning, Memory, and Cognition, 33, 321-334. doi: https://doi.org/10.1037/0278-7393.33.2.321

Roediger, H. L., III, \& McDermott, K. B. (1995). Creating false memories: Remembering words not presented in lists. Journal of
Experimental Psychology: Learning, Memory, and Cognition, 21, 803-814. doi:https://doi.org/10.1037/0278-7393.21.4.803

Rogers, C. S., Jacoby, L. L., \& Sommers, M. S. (2012). Frequent false hearing by older adults: The role of age differences in metacognition. Psychology and Aging, 27, 33-45. doi:https://doi.org/10.1037/ a0026231

Shipley, W. C. (1940). A self-administering scale for measuring intellectual impairment and deterioration. The Journal of Psychology, 9, 371-377. doi:https://doi.org/10.1080/00223980.1940.9917704

Sommers, M. S., \& Huff, L. M. (2003). The effects of age and dementia of the Alzheimer's type on phonological false memories. Psychology and Aging, 18, 791-806. doi:https://doi.org/10.1037/ 0882-7974.18.4.791

Sommers, M. S., Morton, J., \& Rogers, C. (2015). You are not listening to what I said: False hearing in young and older adults. In D. S. Lindsay, C. M. Kelley, A. P. Yonelinas, \& H. L. Roediger III (Eds.), Remembering: Attributions, processes, and control in human memory (essays in Honor of Larry Jacoby) (pp. 269-284). New York, NY: Psychology Press.

Tulving, E. (1985). Memory and consciousness. Canadian Psychology, 26, 1-12. doi:https://doi.org/10.1037/h0080017

Wheeler, M. A., Stuss, D. T., \& Tulving, E. (1997). Toward a theory of episodic memory: The frontal lobes and autonoetic consciousness. Psychological Bulletin, 121, 331-354. doi:https://doi.org/10.1037/ 0033-2909.121.3.331

Publisher's note Springer Nature remains neutral with regard to jurisdictional claims in published maps and institutional affiliations. 